\title{
A PRELIMINARY CHECKLIST OF THE ANGIOSPERMIC FLORA OF DAULATPUR UPAZILA IN KUSHTIA DISTRICT, BANGLADESH
}

\author{
MD. MONIRUZZAMAN, MD. ABUL HASSAN, MD. MIGANUR RAHMAN*, \\ SHEHELEY LAYLA AND MD. RAFIQUL ISLAM \\ Department of Botany, University of Dhaka, Dhaka-1000 Bangladesh
}

\begin{abstract}
A total of 125 angiosperms under 103 genera and 52 families have been recorded from Daulatpur Upazila in Kushtia. Magnoliopsida is represented by 41 families, 73 genera and 86 species, whereas Liliopsida is represented by 11 families, 30 genera and 39 species. In Liliopsida, Poaceae appears to be the largest family comprising 15 genera and 20 species. In Magnoliopsida, Euphorbiaceae appears to be the largest family comprising 9 genera and 14 species.
\end{abstract}

Key words: Angiospermic flora, Daulatpur Upazila, Kushtia

\section{Introduction}

Daulatpur Upazila is located in Kushtia district and lies between $23^{\circ} 52^{\prime}$ and $24^{\circ} 12^{\prime} \mathrm{N}$ latitudes and $88^{\circ} 42^{\prime}$ and $88^{\circ} 58^{\prime} \mathrm{E}$ longitudes. It is bounded on the north by Bagha upazila of Rajshahi district and Lalpur upazila of Natore district, on the east by Mirpur upazila, on the south by Mirpur upazila and Gangni upazila of Meherpur district. The soil mainly calcareous brown floodpain and composed of alluvium and sandy clay with a good percentage of potash and phosphate (Mondal 2003). The area enjoys a tropical climate characterized by a period of high precipitation from June to August and six months of relatively dry period from November to April. The mean annual rainfall is about 1080 $\mathrm{mm}$. Temperature of the area ranges from $21.0-33.7^{\circ} \mathrm{C}$. The maximum temperature was recorded in July and the minimum was recorded in January during the study period (Source: Bangladesh Meteorological Department). This upazila is mainly cultivated area and covers an area of about 468.76 square kilometres. There are fallow land, crop fields, ponds, ditches and beels in the study area.

Daulatpur Upazila consists of medium grade plant diversity. A good number of floristic studies have so far been done in Bangladesh including (Khan and Alam 1996, Uddin and Rahman 1999, Uddin et al. 2003, Tutul et al. 2009, Rahman et al. 2010 and Arefin et al. 2011). No floristic studies are available in Daulatpur Upazila of Kushtia district. Moreover, the area supports many angiospemic species including herbs, shrubs, trees, climbers, epiphytes, parasites and also plenty of hydrophytes. Like other parts of the country, the floristic elements of this area are in risk because of various anthropogenic activities including irrigation, modern agriculture, population settlements, firewood collection and also habitat degradation. In order to make a documentation of the

${ }^{*}$ Dept. of Botany, Mohammadpur Kendriya College, Dhaka-1207, Bangladesh 
angiospermic vegetation of the area, an attempt has been made to prepare a preliminary checklist of the angiospermic plant species occurring in Daulatpur Upazila of Kushtia district.

\section{Materialas and Methods}

The work is based on the fresh materials collected through four field trips to Daulatpur area during 2004 and 2005. Botanical specimens were collected and identified at the department of Botany, University of Dhaka. Identifications were confirmed comparing with herbarium specimens at Dhaka University Salar Khan Herbarium (DUSH) and Bangladesh National Herbarium. In some cases, standard literatures, such as Hooker (1872-1897), Prain (1903), Khan (1977, 1984, 1985) and Uddin and Hassan (2004) were consulted for identification purpose. The families have been arranged according to Cronquist (1981). The specimens were deposited in the Dhaka University Salar Khan Herbarium (DUSH) for future reference.

\section{Results and Discussion}

In the present survey, a total of 125 angiospermic species under 103 genera and 52 families were recorded from Daulatpur Upazila. Magnoliopsida is represented by 41 families, 73 genera and 86 species, while Liliopsida is represented by 11 families, 30 genera and 39 species. Habit wise itemization of plant speices shows that $58.4 \%$ of the total species represented by herbs, $13.6 \%$ by shrubs, $18.4 \%$ by trees, $8.8 \%$ by climbers and $0.8 \%$ by parasites. The genera under each family and the species under each genus are arranged in an alphabetic order. For each species, nomenclature has been brought up to date. Local name (wherever available) and a short annotation are provided.

\section{Magnoliopsida (Dicots)}

1. Annonaceae: Annona reticularia L., Sp. Pl.: 537 (1753). Local names: Nona ata, Nona, Ata. A small tree. Representative specimen: Khalishakundi, 26.09.2004, Md. Moniruzzaman 54 (DUSH).

Annona squamosa L., Sp. Pl.: 537 (1753). Local names: Sharifa, Sitaphal, Maoya. A small tree. Representative specimen: Khalishakundi, 26.09.2004, Md. Moniruzzaman 59 (DUSH).

2. Lauraceae: Litsea glutinosa (Lour.) C.B. Robinson in Philip. J. Sci. Bot. 6: 321 (1911). Sebifera glutinosa Lour., Fl. Cochinch.: 638 (1990). Local names: Chapaitta, Menda, Papoulti. A medium-sized, evergreen tree. Representative specimen: Khalishakundi, 26.09.2004, Md. Moniruzzaman 44 (DUSH).

3. Piperaceae : Peperomia pellucida (L.) H.B.K., Nov. Gen. et. Sp. 1: 64 (1815). Piper pellucidum L., Sp. Pl. 1: 30 (1753). Local names: Peperomia, Luchipata. A small, annual herb. Representative specimen: Udoynagar, 17.11.2004, Md. Moniruzzaman 96 (DUSH). 
Piper longum L., Sp. Pl. 1: 28 (1753). Local names: Pipul, Pipla, Pipla-mul, Pipulmorich. A perennial herb, branched with swollen nodes. Representative specimen: Udoynagar, 17.11.2004, Md. Moniruzzaman 92 (DUSH).

4. Menispermaceae: Stephania japonica (Thunb.) Miers, Ann. Mag. Nat. Hist. Ser. 3, 18: 14 (1866). Menispermum japonicum Thunb., Fl. Jap.: 193 (1784). Local names: Maknadi, Aknadi, Bommokopat. A slender climber. Representative specimen: Udoynagar, 25.09.2004, Md. Moniruzzaman 13 (DUSH).

5. Papaveraceae : Argemone mexicana L., Sp. Pl.: 508 (1753). Local names: Sialkanta, Barosial-kanta. An annual herb. Representative specimen: Udoynagar, 24.01.2005, Md. Moniruzzaman 156 (DUSH).

6. Ulmaceae: Trema orientalis (L.) Bl., Mus. Bot. Lugd.-Bat. 2: 63 (1856). Celtis orientalis L., Sp. Pl.: 120044 (1753). Local names: Jilan, Narsa, Gobra. An evergreen small tree. Representative specimen: Khalishakundi, 26.09.2004, Md. Moniruzzaman 66 (DUSH).

7. Moraceae: Artocarpus chaplasha Roxb., Fl. Ind. 3: 525 (1832). Local name: Chapalish. A lofty deciduous tree. Representative specimen: Khalishakundi, 26.09.2004, Md. Moniruzzaman 39 (DUSH).

Ficus benghalensis L., Sp. Pl.: 1059 (1753). Local name: Bot. A large, spreading tree. Representative specimen: Shampur, 10.06.2005, Md. Moniruzzaman 219 (DUSH).

Ficus heterophylla L. f., Suppl.: 442 (1781). Local name: Bhuidumur. A hispid, scandent shrub. Representative specimen: Khalishakundi, 17.11.2004, Md. Moniruzzaman 97 (DUSH).

Ficus hispida L. f., Suppl. Pl.: 442 (1781). Local names: Kakdumur, Dumur, Khukshidumur. A small tree. Representative specimen: Khalishakundi, 26.09.2004, Md. Moniruzzaman 49 (DUSH).

Ficus religiosa L., Sp. Pl.: 1059 (1753). Local names: Ashathwa, Pipal. A large tree. Representative specimen: Khalishakundi, 17.11.2004, Md. Moniruzzaman 100 (DUSH).

8. Urticaceae: Laportea interrupta L., Sp. Pl.: 985 (1753). Local name: Lal Bichuti. An erect annual herb. Representative specimen: Khalishakundi, 26.09.2004, Md. Moniruzzaman 11 (DUSH).

9. Nyctaginaceae: Boerhavia diffusa L., Sp. Pl. 1: 3 (1753). Local names: Punarnava, Gondhapurna. A perennial herb. Representative specimen: Daulatpur, 27.09.2004, Md. Moniruzzaman 88 (DUSH).

10. Chenopodiaceae : Chenopodium ambrosioides L., Sp. Pl.: 219 (1753). An annual, erect herb. Representative specimen: Barogandia, 24.01.2005, Md. Moniruzzaman 155 (DUSH). 
11. Amaranthaceae : Achyranthes aspera L., Sp. Pl. 1: 204 (1753). Local name: Apang. A perennial herb. Representative specimen: Barogandia, 26.09.2004, Md. Moniruzzaman 58 (DUSH).

Alternanthera philoxeroides (Mart.) Griseb., Abh. Ges. Goett. Wiss 24: 36 (1879). Bucholzia phyloxeroides Mart., Beitr. Amar.: 107 (1825). Local name: Helencha. An annual herb. Representative specimen: Barogandia, 26.09.2004, Md. Moniruzzaman 65 (DUSH).

Alternanthera sessilis (L.) DC., Cat. Pl. Hort. Monspel.: 77 (1813). Gomphrena sessilis L., Sp. Pl.: 225 (1753). Local names: Chanchi, Haicha, Sachisak. A herb, usually decumbent or prostrate. Representative specimen: Boalia, 26.09.2004, Md. Moniruzzaman 21 (DUSH).

Amaranthus spinosus L., Sp. Pl. 1: 991 (1753). Local names: Kanta-note, Kantadenga, Katamiris. An annual, erect, spinescent herb. Representative specimen: Daulatpur, 25.09.2004, Md. Moniruzzaman 19 (DUSH).

Amaranthus viridis L., Sp. Pl. ed. 2: 1405 (1753). Local names: Note sak, Marissag. An annual, erect or decumbent, small, slender herb. Representative specimen: Daulatpur, 25.01.2005, Md. Moniruzzaman 186 (DUSH).

12. Polygonaceae : Polygonum plebejum R. Br., Prodr. Fl. Nov. Holl.: 420 (1810). A prostrate or diffuse herb. Representative specimen: Malipara, 25.01.2005, Md. Moniruzzaman 165 (DUSH).

Rumex maritimus L., Sp. Pl.: 335 (1753). Local name: Ban palang. An annual herb. Representative specimen: Barogandia, 24.01.2005, Md. Moniruzzaman 145 (DUSH).

13. Bombacaceae : Bombax ceiba L., Sp. Pl.: 511 (1753). Local names: Shimul tula, Pakra, Tula gachh. A large tree with buttress base. Representative specimen: Malipara, 26.09.2004, Md. Moniruzzaman 43 (DUSH).

14. Malvaceae : Urena lobata L., Sp. Pl.: 692 (1753). Local name: Banokra. An undershrub. Representative specimen: Malipara, 26.09.2004, Md. Moniruzzaman 16 (DUSH).

15. Passifloraceae : Passiflora foetida L., Sp. Pl.: 959 (1753). Local names: Jhumkolata, Gorakphul. A climbing slender shrub. Representative specimen: Malipara, 27.09.2004, Md. Moniruzzaman 82 (DUSH).

16. Cucurbitaceae : Coccinia grandis (L.) Voit., Hort. Suburb. Calcut.: 59 (1845). Bryonia grandis L., Mant. P1. 1: 126 (1767). Local names: Kawajhinga, Telakucha. A much branched, climbing or prostrate herb. Representative specimen: Khalishakundi, 17.11.2004, Md. Moniruzzaman 101 (DUSH).

17. Moringgaceae : Moringa oleifera Lam., Encycl. 1 (2): 398 (1785). Local names: Sajna, Sojne. A small tree. Representative specimen: Goalgram, 17.11.2004, Md. Moniruzzaman 94 (DUSH). 
18. Primulaceae : Anagallis arvensis L., Sp. Pl.: 148 (1753). Erect or decumbentascending; glabrous-dotted herbs. Representative specimen: Goalgram, 25.01.2005, Md. Moniruzzaman 159 (DUSH).

19. Mimosaceae: Acacia nilotica (L.) Delile subsp. indica (Benth.) Brenan in Kew Bull. 12: 84 (1957). Mimosa nilotica L., Sp. Pl.: 152 (1753). Local names: Babla, Babul. A tree. Representative specimen: Khalishakundi, 09.06.2005, Md. Moniruzzaman 194 (DUSH).

20. Caesalpiniaceae : Senna tora (L.) Roxb., Fl. Ind. 2: 340 (1832). Cassia tora L., Sp. Pl: 376 (1753). Local name: Chakunda. A perennial, erect, foetid, often profusely branched herb or undershrub. Representative specimen: Udoynagar, 26.09.2004, Md. Moniruzzaman 51 (DUSH).

21. Fabaceae : Desmodium heterophyllum (Willd.) DC., Prodr. 2: 334 (1825). Desmodium triflorum Wight. \& Arn., Prodr.: 229 (1834). A procumbent herb. Representative specimen: Udoynagar, 26.09.2004, Md. Moniruzzaman 42 (DUSH).

Erythrina variegata L., in stickm. Herb. Amboin.10 (1754). Local names: Mandar, Madar, Plitamadar, Paniamadar. A deciduous, prickly tree. Representative specimen: Udoynagar, 26.09.2004, Md. Moniruzzaman 45 (DUSH).

22. Onagraceae: Ludwigia hyssopifolia (G. Don) Exell., Garica de Orta 5: 471 (1957). Jussiaea hyssopifolia G. Don, Gen. Syst. 2: 693 (1832). A branched herb. Representative specimen: Udoynagar, 17.11.2004, Md. Moniruzzaman 104 (DUSH).

23. Euphorbiaceae: Acalypha indica L., Sp. Pl.: 1003 (1753). Local name: Muktajhuri. A small robust or woody herb. Representative specimen: Udoynagar, 26.09.2004, Md. Moniruzzaman 60 (DUSH).

Chrozophora plicata Hook. f., Fl. Br. Ind. 5: 409 (1887). An annual herb. Representative specimen: Daulatpur, 09.06.2005, Md. Moniruzzaman 200 (DUSH).

Croton bonplandianus Bill., Adansonia 4: 339 (1864). Local name: Moricha. An annual herb. Representative specimen: Boalia, 25.01.2005, Md. Moniruzzaman 184 (DUSH).

Euphorbia antiquorum L., Sp. Pl.: 42005 (1753). An erect, trunk straight tree. Representative specimen: Boalia, 25.01.2005, Md. Moniruzzaman 84 (DUSH).

Euphorbia heyneana Sprengel, Syst. Veg. 3: 791 (1826) Local name: Chhota Kerui. An annual herb. Representative specimen: Udoynagar, 25.09.2004, Md. Moniruzzaman 10 (DUSH).

Euphorbia hirta L., Sp. Pl.: 454 (1753). Local names: Dudhia, Ghaopata, Baradudhi. An annual, erect or ascending herb. Representative specimen: Boalia, 26.09.2004, Md. Moniruzzaman 33 (DUSH).

Euphorbia tithymaloides L., Sp. Pl.: 453 (1753). Local name: Rangchita. A subsucculent, shrub. Representative specimen: Daulatpur, 22.09.2004, Md. Moniruzzaman 91 (DUSH). 
Jatropha curcas L., Sp. Pl.: 1006 (1753). Local name: Veron. A large, glabrous shrub or rarely small tree. Representative specimen: Daulatpur, 27.09.2004, Md. Moniruzzaman 85 (DUSH).

Jatropha gossypifolia L., Sp. Pl.: 1006 (1753). Local names: Lalbherenda, Laljeol. A small shrub. Representative specimen: Daulatpur, 26.09.2004, Md. Moniruzzaman 31 (DUSH).

Phyllanthus fraternus Webster, Contr. Gray Herb. 176: 53 (1955). Phyllanthus niruri senu Hook. f., Fl. Brit. Ind. 5: 298 (1887). Local name: Bhui amla. A herb. Representative specimen: Khalishakundi, 17.11.2004, Md. Moniruzzaman 108 (DUSH).

Phyllanthus reticulatus Poir. in Lamk., Encycl. Meth. B. 5: 298 (1804). Local name: Panseuli. A large, scandent shrub. Representative specimen: Khalishakundi, 17.11.2004, Md. Moniruzzaman 184 (DUSH).

Phyllanthus urinaria L., Sp. Pl.: 982 (1753). An erect, glabrous, annual herb. Representative specimen: Daulatpur, 25.09.2004, Md. Moniruzzaman 5 (DUSH).

Ricinus communis L., Sp. Pl.: 1007 (1753). Local name: Rerhi. An evergreen shrub. Representative specimen: Daulatpur, 24.01.2005, Md. Moniruzzaman 124 (DUSH).

Trewia nudiflora L., Sp. Pl. ed. 3: 166 (1753). Local names: Lattu, Pitali, Latim. A deciduous tree. Representative specimen: Khalishakundi, 26.09.2004, Md. Moniruzzaman 48 (DUSH).

24. Vitaceae: Cayratia trifolia (L.) Domin, Biblioth. Bot. 89: 371 (1927). Vitis trifolia L., Sp. Pl.: 203 (1753). Local name: Amal-lata. A slender, herbaceous climber with swollen rootstock. Representative specimen: Udoynagar, 26.09.2004, Md. Moniruzzaman 61 (DUSH).

25. Anacardiaceae : Spondias pinnata (L. f.) Kurz, Pegu Rep. A. 44 (1875). Local name: Piyala. A medium-sized to tall tree. Representative specimen: Udoynagar, 26.09.2004, Md. Moniruzzaman 68 (DUSH).

26. Meliaceae: Melia azedarach L., Sp. Pl.: 384 (1753). Local name: Gora nim. A medium-sized tree. Representative specimen: Daulatpur, 26.09.2004, Md. Moniruzzaman 67 (DUSH).

27. Rutaceae : Aegle marmelos (L.) Corr., Trans. Linn. Soc. 5: 222 (1800). Crateva marmelos L., Sp. Pl.: 444 (1753). Local name: Bel. A small, deciduous tree. Representative specimen: Daulatpur, 27.09.2004, Md. Moniruzzaman 81 (DUSH).

Glycosmis pentaphylla (Retz.) A. DC., Prodr. 1: 538 (1824). Limonia pentaphylla Retz., Obs. Bot. 5: 24 (1788). Local names: Aisshara, Matmati, Datmajon. A shrub or small tree. Representative specimen: Daulatpur, 26.09.2004, Md. Moniruzzaman 50 (DUSH).

28. Oxalidaceae : Oxalis corniculata L., Sp. Pl.: 435 (1753). Local name: Ambuli. An annual herb. Representative specimen: Goalgram, 26.09.2004, Md. Moniruzzaman 32 (DUSH). 
29. Apiaceae: Centella asiatica (L.) Urban in Mart., Fl. Bras. 11: 287 (1879). Hydrocotyle asiatica L., Sp. Pl. 1: 234 (1753). Local names: Thankuni, Adamoni. A perennial herb. Representative specimen: Goalgram, 24.01.2005, Md. Moniruzzaman 146 (DUSH).

30. Asclepiadaceae: Asclepias gigantea L., Sp. Pl.: 214 (1753). Local name: Akanda. A small shrub. Representative specimen: Goalgram, 26.09.2004, Md. Moniruzzaman 26 (DUSH).

31. Solanaceae: Datura metel L., Sp. Pl.: 179 (1753). Local names: Dutra, Dutura. A stout herb. Representative specimen: Malipara, 27.09.2004, Md. Moniruzzaman 90 (DUSH).

Nicotiana plumbaginifolia Viv., Elench. Pl. Hort. Dinergo: 26. t. 5 (1802). Local name: Tamak. A slender, erect, annual herb. Representative specimen: Malipara, 09.06.2005, Md. Moniruzzaman 195 (DUSH).

Physalis minima L., Sp. Pl.: 183 (1753). Annual glabrous herb. Representative specimen: Daulatkhali, 17.11.2004, Md. Moniruzzaman 115 (DUSH).

Solanum nigrum L., Sp. Pl.: 186 (1753). Local names: Tit begun, Puti begun. An annual erect shrub. Representative specimen: Daulatkhali, 17.11.2004, Md. Moniruzzaman 113 (DUSH).

32. Convolvulaceae : Ipomoea aquatica Forssk., Fl. Aeg.-Arab.: 44 (1775). Local name: Kalmilata. A glabrous trailer on water. Representative specimen: Daulatkhali, 25.01.2005, Md. Moniruzzaman 176 (DUSH).

Ipomoea fistulosa Mart. ex Choisy in DC., Prodr. 9: 349 (1845). Local names: Dholkalmi, Durakalma. A fistular shrub. Representative specimen: Daulatkhali, 25.01.2005, Md. Moniruzzaman 190 (DUSH).

33. Cuscutaceae : Cuscuta reflexa Roxb., Pl. Corom. 2: 3, t. 104 (1798). Local names: Swarnalata, Jarbuti, Algusi. A fleshy parasite, forming dense yellow masses on small tree or shrub. Representative specimen: Daulatkhali, 17.11.2004, Md. Moniruzzaman 118 (DUSH).

34. Boraginaceae : Heliotropium indicum L., Sp. Pl.: 130 (1753). Local name: Hatisur. An annual herb. Representative specimen: Daulatkhali, 10.06.2005, Md. Moniruzzaman 215 (DUSH).

Cordia dichotoma Forst. f., Fl. Ins. Auster. Proder, 18: 110 (1876). Local name: Boula. A shrub or small tree. Representative specimen: Daulatpur, 26.09.2004, Md. Moniruzzaman 87 (DUSH).

35. Verbenaceae : Callicarpa longifolia Lamk., Enc. Meth. 1: 403 (1798). Local name: Bormala. A shrub. Representative specimen: Daulatpur, 26.09.2004, Md. Moniruzzaman 87 (DUSH). 
Lippia alba (Mill). Briton et Wilson. Sci. Surv. Puerto Rico. Vergin 6: 141 (1935). An undershrub. Representative specimen: Daulatpur, 26.09.2004, Md. Moniruzzaman 20 (DUSH).

36. Lamiaceae : Anisomeles indica (L.) O. Kuntze, Rev. Gen.: 512 (1891). Nepeta indica L., Sp. Pl.: 596 (1753). Local name: Gobura. A bushy undershrub. Representative specimen: Daulatpur, 17.11.2004, Md. Moniruzzaman 117 (DUSH).

Dysophylla crassicaulis Benth. in Wall., Pl. As. Rar. 1: 30 (1830). An annual herb. Representative specimen: Malipara, 27.09.2004, Md. Moniruzzaman 91 (DUSH).

Hyptis suaveolens (L.) Poit., Ann. Mus. Par. 7: 472, t. 29 (1806). Ballota suaveolens L., Syst. Nat. ed. 10: 1100 (1759). Local name: Tokma. An annual herb. Representative specimen: Malipara, 25.01.2005, Md. Moniruzzaman 188 (DUSH).

37. Scrophulariaceae : Lindernia crustacea (L.) F. Muell., Cens. Austr. Pl.: 97 (1882). Capraria crustacea L. Mant. Pl. 1: 87 (1767). A dichotomously branched, prostrate herb. Representative specimen: Khalishakundi, 25.09.2004, Md. Moniruzzaman 6 (DUSH).

Scoparia dulcis L., Sp. Pl.: 166 (1753). Local name: Bondhuna. A herb. Representative specimen: Daulatpur, 25.09.2004, Md. Moniruzzaman 8 (DUSH).

38. Orobanchaceae: Orobanche aegyptiaca Pers., Syn. Pl. 2: 181 (1807). Local names: Poramula, Misridana. A herb. Representative specimen: Daulatpur, 25.01.2005, Md. Moniruzzaman 160 (DUSH).

39. Acanthaceae: Hygrophila salicifolia (Vahl) Nees in Wall., Pl. As. Rar. 3: 81 (1832). Ruellia salicifolia Vahl, Symb. 3: 84 (1794). A prostrate to erect herb. Representative specimen: Daulatpur, 25.01.2005, Md. Moniruzzaman 120 (DUSH).

Rungia pectinata (L.) Nees in DC., Prodr. 11: 469 (1847). Justicia pectinata L., Amoen. Acad. 4: 293 (1759). Local name: Pindi. A much branched, prostrate or suberect herb. Representative specimen: Daulatpur, 17.11.2004, Md. Moniruzzaman 120 (DUSH).

40. Rubiaceae: Neolamarckia cadamba (Roxb.) Bosser, Bull. Mus. Hist. Nat. Paris, Ser. 6, Sec. B. Adans. 3: 247 (1984). Local name: Kadam. A deciduous tree. Representative specimen: Daulatpur, 17.11.2004, Md. Moniruzzaman 103 (DUSH).

Hedyotis scandens Roxb., Fl. Ind. 1: 369 (1820). A climbing shrub. Representative specimen: Daulatpur, 24.01.2005, Md. Moniruzzaman 134 (DUSH).

41. Asteraceae: Ageratum conyzoides L., Sp. Pl.: 839 (1753). Local names: Fulkuri, Hialmuti. An annual herb. Representative specimen: Daulatpur, 17.11.2004, Md. Moniruzzaman 109 (DUSH).

Eclipta prostrata L., Mant. 2: 286 (1771). Local name: Kalokeshoti. An erect, annual herb. Representative specimen: Udoynagar, 24.01.2005, Md. Moniruzzaman 142 (DUSH). 
Enhydra fluctuans Lour., Fl. Cochinch.: 511 (1790). Local name: Helencha. A profusely branched, annual aquatic herb. Representative specimen: Udoynagar, 27.09.2004, Md. Moniruzzaman 80 (DUSH).

Gnaphalium luteo-album L., subsp. affine (D. Don) Koster, Blumea 4: 484 (1941). An erect, annual herb. Representative specimen: Daulatpur, 25.01.2005, Md. Moniruzzaman 170 (DUSH).

Grangea maderaspatana (L.) Poir., Enc. Suppl. 2: 825 (1811). Artemisia maderaspatana L., Sp. Pl.: 849 (1753). An annual herb. Representative specimen: Goalgram, 24.01.2005, Md. Moniruzzaman 139 (DUSH).

Mikania cordata (Burm. f.) B. L. Robinson, Contrib. Gray Herb. 104: 65 (1934). Eupatorium cordatum Burm. f., Fl. Ind.: 176 (1768). Local names: Asamlata, Tarulata. A perennial herb. Representative specimen: Daulatkhali, 26.09.2004, Md. Moniruzzaman 17 (DUSH).

Spilanthes calva DC. in Wight, Contrib. Bot. Ind.: 19 (1834). An annual herb. Representative specimen: Khalishakundi, 26.09.2004, Md. Moniruzzaman 30 (DUSH).

Synedrella nodiflora (L.) Gaertn., Fruct. 2: 456, t. 171 (1791). Verbesina nodiflora L., Cent. Pl. 1: 28 (1755). A small woody herb. Representative specimen: Daulatpur, 26.09.2004, Md. Moniruzzaman 71 (DUSH).

Xanthium indicum Koen. ex Roxb., Fl. Ind. 3: 601 (1832). An erect, annual herb. Representative specimen: Daulatpur, 25.01.2005, Md. Moniruzzaman 187 (DUSH).

\section{Liliopsida (Monocots)}

42. Alismataceae : Sagittaria sagittifolia L., Sp. Pl. 2: 993 (1753). Local names: Chhotokut, Muyamuya. A scapigerous herb. Representative specimen: Daulatpur, 25.01.2005, Md. Moniruzzaman 180 (DUSH).

43. Arecaceae : Phoenix sylvestris (L.) Roxb., Hort. Beng. 73 (1814). Local name: Khejur. A tall palm tree. Representative specimen: Khalishakundi, 26.09.2004, Md. Moniruzzaman 24 (DUSH).

44. Araceae : Alocasia fornicata (Roxb.) Schott, Oestr. Bot. Wochenbl. 4: 410 (1854). Arum fornicatum Roxb., Fl. Ind. 3: 501 (1832). A tuberous, coarse herb. Representative specimen: Khalishakundi, 10.06.2005, Md. Moniruzzaman 217 (DUSH).

Colocasia esculenta (L.) Schott in Schott \& Endl., Melet. Bot.: 18 (1832). Arum esculentum L., Sp. Pl.: 965 (1753). Local name: Kachu. A tall coarse herb. Representative specimen: Khalishakundi, 27.09.2004, Md. Moniruzzaman 83 (DUSH).

Typhonium trilobatum (L.) Schott., Wien. Zeitschr. 3: 72 (1829). Arum trilobatum L., Sp. Pl.: 934 (1753). A tuberous climber. Representative specimen: Khalishakundi, 10.06.2005, Md. Moniruzzaman 223 (DUSH). 
45. Commelinaceae : Commelina benghalensis L., Sp. Pl.: 41 (1753). Local names: Dholpata, Kanchra. A slender herb. Representative specimen: Malipara, 25.09.2004, Md. Moniruzzaman 2 (DUSH).

Murdania nudiflora (L.) Brenan, Kew Bull. 7: 189 (1952). Commelina nudiflora L., Sp. Pl.: 41 (1753). An annual, diffuse herb. Representative specimen: Malipara, 25.09.2004, Md. Moniruzzaman 3 (DUSH).

46. Cyperaceae : Cyperus compressus L., Sp. Pl. ed. 1: 46 (1753). An annual herb, tufted root. Representative specimen: Daulatpur, 25.01.2005, Md. Moniruzzaman 204 (DUSH).

Cyperus difformis L., Cent. Pl. 2: 6 (1756). Local name: Mutha ghas. An annual, tufted herb. Representative specimen: Taragunia, 10.01.2005, Md. Moniruzzaman 213 (DUSH).

Cyperus diffusus Vahl, Enum. Pl. 2: 321 (1806). A perennial herb. Representative specimen: Taragunia, 24.01.2005, Md. Moniruzzaman 140 (DUSH).

Cyperus michelianus (L.) Link., Hort. Bot. Berol. Descr. 1: 303 (1827). Scirpus michelianus L., Sp. Pl.: 45 (1753). Local name: Choto Gotubi. An annual herb. Representative specimen: Taragunia, 25.01.2005, Md. Moniruzzaman 173 (DUSH).

Cyperus rotundus L., Sp. Pl.: 45 (1753). Local name: Motha ghas. Perennial grass. Representative specimen: Daulatpur, 17.11.2004, Md. Moniruzzaman 187 (DUSH).

47. Poaceae : Axonopus compressus (Sw.) P. Beauv., Ess. Agrost. 12 (154): 167 (1812). Milium compressum Sw., Prod.: 24 (1788). A perennial, tufted herb. Representative specimen: Moubaria, 17.11.2004, Md. Moniruzzaman 98 (DUSH).

Brachiaria distachya (L.) Stapf. In Prain,Fl. Trop. Afr. 9: 565(1919). An annual or perennial grass. Representative specimen: Daulatpur, 26.09.2004, Md. Moniruzzaman 18 (DUSH).

Chrysopogon aciculatus (Retz.) Trin., Fund. Agrost.: 188 (1820). Andropogon aciculatus Retz., Obs. Bot. 5: 22 (1989). A glabrous herb. Representative specimen: Khalishakundi, 26.09.2004, Md. Moniruzzaman 01 (DUSH).

Cynodon dactylon (L.) Pers., Syn. Pl. ed. 1: 85 (182005). Panicum dactylon L., Sp. Pl.: 58 (1753). Local names: Durba, Dubla, Dubraghas. A creeping herb. Representative specimen: Khalishakundi, 17.11.2004, Md. Moniruzzaman 114 (DUSH).

Dactyloctenium aegyptium (L.) P. Beauv., Ess. Agrost. Expl. Pl.: 15 (1812). Cynosurus aegyptius L., Sp. Pl. ed. 1, 1: 72 (1753). Stoloniferous, annual or short-lived perennial herb. Representative specimen: Daulatpur, 26.09.2004, Md. Moniruzzaman 39 (DUSH).

Dichanthium annulatum (Forsskal) Stapf. In Prain,Fl. Trop. Afr. 9: 178 (1917). Local name: Loari. An annual or perennial herb. Representative specimen: Daulatpur, 24.01.2005, Md. Moniruzzaman 123 (DUSH). 
Digitaria ciliaris (Retz.) Koeler, Dese. Gram. 27. (1802) Annual with weak, branching culms. Representative specimen: Daulatpur, 17.11.2004, Md. Moniruzzaman 126 (DUSH).

Digitaria ischaemum (Schreb.) Schreb. ex. Muhl., Descr. Gram. Calam. 131 (1817). An annual, erect or geniculately ascending. Representative specimen: Daulatpur, 09.06.2005, Md. Moniruzzaman 205 (DUSH).

Digitaria longiflora (Retz.) Pers., Syn. Pl. 1: 85 (182005). An annul with slender. Representative specimen: Daulatpur, 10.06.2005, Md. Moniruzzaman 208 (DUSH).

Digitaria sanguinalis (L.) Scop., Flora. Carn. Ed. 2, 1: 52 (1772). An annual or perennial grass. Representative specimen: Daulatpur, 26.09.2004, Md. Moniruzzaman 29 (DUSH).

Echinochloa colona (L.) Link., Hort. Berol. 2: 209 (1833). Local name: Shyamaghas. A leafy annual grass. Representative specimen: Udoynagar, 26.09.2004, Md. Moniruzzaman 69 (DUSH).

Echinochloa cruss-galli (L.) P. Beauv., Ess. Agrost. 53: 161 (1812). Panicum crus-galli L., Sp. Pl. ed. 1, 1: 56 (1753). Local names: Barashyamaghas, Malanga-kuri, Kakligash. An annual or perennial herb. Representative specimen: Udoynagar, 10.06.2005, Md. Moniruzzaman 207 (DUSH).

Eleusine indica (L.) Gaertn., Fruct. 1: 8 (1789). Cynusurus indicus L., Sp. Pl. ed.1.: 72 (1753). Local name: Malankuri. A tufted, annual herb. Representative specimen: Khalishakundi, 25.09.2004, Md. Moniruzzaman 14 (DUSH).

Eragrostis tenella (L.) P. Beauv. ex . Rearn. \& Schult., Syst. Veg. 2: 576 (1817). Local name: Koni. A loosely tufted annual or short lived perennial herb. Representative specimen: Daulatpur, 26.09.2004, Md. Moniruzzaman 46 (DUSH).

Eragrostis unioloides (Retz.) Nees ex Steud., Syn. Pl. Glum. 1: 264 (1854). Poa unioloides Retz. Obs. Bot. 5: 19 (1789). An annual herb. Representative specimen: Daulatpur, 17.11.2004, Md. Moniruzzaman 93 (DUSH).

Hemarthria protensa Steud., Syn. Pl. Glum. 1: 359 (1854). Local name: Chailla. An erect to decumbent herb. Representative specimen: Khalishakundi, 09.06.2005, Md. Moniruzzaman 199 (DUSH).

Oplismenus compositus (L.) P. Beauv., Ess. Agrost. 54: 168 (1812). Panicum compositum L., Sp. Pl. ed. 1: 57 (1753). A perennial grass. Representative specimen: Khalishakundi, 25.09.2004, Md. Moniruzzaman 09 (DUSH).

Panicum repens L., Sp. Pl. ed. 2: 87 (1762). A perennial, rhizomatous grass. Representative specimen: Daulatpur, 10.06.2005, Md. Moniruzzaman 210 (DUSH).

Paspalum scrobiculatum L., Mant. 1: 29 (1767). Local name: Goicha. An annual herb. Representative specimen: Daulatpur, 10.06.2005, Md. Moniruzzaman 211 (DUSH). 
Saccharum spontaneum L., Mant. Pl. 2: 183 (1771). Local name: Kash. A perennial, tall herb. Representative specimen: Daulatpur, 26.09.2004, Md. Moniruzzaman 27 (DUSH).

48. Zingiberaceae : Alpinia nigra (Gaertn.) Burtt., Notes Roy. Bot. Gard. Edinb. 35: 213 (1977). Zingiber nigrum Gaertn. (1788). Local name: Tara. Stem leafy, leaves sessile or sub-sessile. Representative specimen: Daulatpur, 26.09.2004, Md. Moniruzzaman 63 (DUSH).

49. Pontederiaceae : Eichhornia crassipes (Mart.) Solms in A. DC., Mon. Phan. 4: 527 (1883). Pontederia crassipes Mart., Nov. Gen. Sp.: 9, t. 4 (1823). Local names: Kachuripana, Jarmani. An aquatic, free-floating herb. Representative specimen: Goalgram, 10.06.2005, Md. Moniruzzaman 212 (DUSH).

Monochoria hastata (L.) Solms. in A. DC., Mon. Phan. 4: 523 (1883). Pontederia hastata L., Sp. Pl.: 288 (1753). An aquatic, emergent herb. Representative specimen: Goalgram, 09.06.2005, Md. Moniruzzaman 202 (DUSH).

50. Costaceae : Costus speciosus (Koen.) Smith, Trans. Linn. Soc. London 1: 249 (1791). Banksea speciosa Koen. in Retz., Obs. Bot. 3: 75 (1783). Local name: Jongliphul. A rhizomatous herb. Representative specimen: Daulatpur, 26.09.2004, Md. Moniruzzaman 73 (DUSH).

51. Dioscoreaceae : Dioscorea alata L., Sp. P1.: 1033 (1753). Local name: Chupri alu. A perennial climber. Representative specimen: Daulatpur, 26.09.2004, Md. Moniruzzaman 26 (DUSH).

Dioscorea kamoonensis Kunth, Enum. Pl. 5: 395 (1850). Local names: Tepata alu,Erabera lata. A climber. Representative specimen: Daulatpur, 26.09.2004, Md. Moniruzzaman 28 (DUSH).

52. Orchidaceae : Vanda tessellata (Roxb.) Hook. f. ex G. Don in Loud., Hort. Brit.: 372 (1830). Epidendrum tessellatum Roxb., Pl. Corom. 1: 34, t. 42 (1795). An epiphytic herb. Representative specimen: Khalishakundi, 09.06.2005, Md. Moniruzzaman 192 (DUSH).

\section{References}

Arefin M.K., M. M. Rahman, M. Z. Uddin and M. A. Hassan, 2011. Angiosperm flora of Satchari National Park, Habiganj, Bangladesh. Bangladesh J. Plant. Taxon. 18(2): pp. 117-140 .

Bangladesh National Herbarium, Bangladesh Agricultural Research Council, Dhaka.

Cronquist, A. 1981. An integrated system of classification of flowering plants. Columbia University Press, New York, 1262 pp.

Hooker, J.D. 1872-1897. The Flora of British India, Vols. 1-7 (Ind. Repr. 1973). Bishen Singh Mahendra Pal Singh, Dehra Dun, India.

Khan, M.S. 1977. Onagraceae. In: Khan, M.S. (ed.). Flora of Bangladesh. Fasc. 6: 1-10. Bangladesh.

Khan, M.S. 1984. Dipterocarpaceae. In: Khan, M.S. (ed.). Flora of Bangladesh. Fasc. 25: 1-15.

Khan, M.S. 1985. Convolvulaceae. In: Khan, M.S. (ed.). Flora of Bangladesh. Fasc. 30: 1-59. 
Khan, M.S. and M. Alam 1996. Homestead Flora of Bangladesh. Bangladesh Agricultural Research Council, Dhaka. 275 pp.

Mondal, R. 2003. In: Sirajul Islam (ed.). Banglapedia. Asiatic Society of Bangladesh. Dhaka 9:345 pp.

Prain, D. 1903. Bengal Plants. Vols. 1 \& 2 (Ind. Repr. 1981). Bishen Singh Mahendra Pal Singh, Dehra Dun, India.

Rahman, M. O., M.Z. Uddin, E. Tutul, M. Begum and M. A. Hassan, 2010. Additions to the Angiopermic Flora of Runctia Sal Forest, Bangladesh. Bangladesh J. Plant Taxon. 17(2): pp. 167-181.

Tutul E., M. Z. Uddin, M. O. Rahman and M. A. Hassan, 2009. Angiopermic Flora of Runctia Sal Forest, Bangladesh. 1. Liliopsida (Monocots). Bangladesh J. Plant Taxon. 16: pp. 8390.

Uddin, S. B. and M. A. Rahman, 1999. Angiospermic flora of Himchari National Park, Cox's Bazar. Bangladesh J. Plant Taxon. 6(1): pp. 31-68.

Uddin, M. Z., M. A. Hassan and M. S. Khan, 2003. An annotated checklist of angiospermic flora of Rema-Kalenga Wildlife Sanctuary (Habiganj) In Banglades-II (a): Magnoliopsida (Dicots). Bangladesh J. Plant Taxon. 10(1): pp. 79-94.

Uddin, M.Z. and M.A. Hassan. 2004. Flora of Rema-Kalenga Wildlife Sanctuary. IUCN Bangladesh Country Office, Dhaka, Bangladesh, vi + 120 pp.

(Received revised manuscript on 30 May 2012) 